Il est prouvé que la nicotine est une source de dépendance. Le tabagisme provoque non seulement le cancer, mais aussi d'autres maladies mortelles et invalidantes, telles que maladie coronaire et BPCO. A tout âge, fumer a des conséquences néfastes, du poids défectueux si on fume pendant une grossesse à l'artériosclérose à évolution invalidante, pouvant entraîner l'amputation d'une jambe suite au vieillissement précoce du fumeur. Un fumeur sur deux meurt prématurément des conséquences de sa dépendance. Et désormais ce sont les enfants, les jeunes et surtout les femmes que les géants du tabac ont dans le viseur.

Si les tendances actuelles en matière de dépendance à la cigarette devaient se poursuivre, le nombre de personnes décédées des suites du tabagisme atteindrait un milliard au cours de ce siècle. Et chaque décennie ferait cent millions de personnes de victimes à partir de l'an 2050.
En Occident, les Etats américains de Californie et du Massachusetts sont les premiers à avoir démontré que des efforts constants et systématiques pour lutter contre le tabagisme dans les lieux publics pouvaient entraîner une nette réduction de la consommation de cigarettes et qu'il était possible de stabiliser ces résultats. Avec les moyens et les mesures politiques d'accompagnement nécessaires, un tel succès est aussi réalisable chez nous.

En tant que spécialistes, nous portons une grande responsabilité puisque le tabagisme est la principale cause de maladies mortelles évitables dans notre pays.

Dr Thomas Cerny, professeur, président de la Ligue suisse contre le cancer

\title{
Résolution pour un avenir sans tabac
}

\author{
Conférence nationale sur la prévention du tabagisme des $1^{\text {er }}$ et 2 décembre 2004 à Fribourg
}

\section{Pas à pas vers un avenir sans tabac}

Chaque jour, plus de 20 personnes meurent prématurément en Suisse des effets du tabagisme, soit plus de 8000 décès par an, dont quelques centaines de victimes de la fumée passive. 45 pour cent de ces décès sont causés par des maladies cardiovasculaires dues au tabagisme, 25 pour cent par le cancer du poumon, 18 pour cent par des maladies des voies respiratoires et 12 pour cent par d'autres types de cancer.

Les participants à la Conférence nationale sur la prévention du tabagisme des $1^{\mathrm{er}}$ et 2 décembre 2004 à Fribourg préconisent donc les mesures suivantes:

\section{Protection contre la fumée passive}

Les personnes qui veulent vivre sans tabac ne sont protégées efficacement de la fumée passive que dans des locaux absolument exempts de fumée. L'époque des réglementations à la carte et de la mise à disposition de simples coins sans fumée appartient au passé.

C'est pourquoi nous saluons l'initiative politique du Conseil d'Etat tessinois de rendre tous les lieux publics sans fumée. Nous invitons les autres cantons à garantir aussi la protection contre la fumée passive dans la loi.

Le droit à une place de travail sans fumée doit s'appliquer sans restriction. Il convient d'adapter en conséquence l'ordonnance 3 relative à la loi du travail. Les inspectorats cantonaux du travail doivent exercer leur fonction de contrôle.

\section{Soutien en cas d'arrêt du tabagisme}

La majorité des fumeurs souhaitent se débarrasser de leur dépendance à la nicotine. Les mesures prises dans le cadre de la campagne nationale «Fumer, ça fait du mal - Let it be» doivent être poursuivies: médecins, équipes des cabinets dentaires et des pharmacies, centres cantonaux de prévention doivent être habilités et motivés à conseiller et soutenir les fumeurs qui souhaitent arrêter.

Il importe que le travail de ces spécialistes soit honoré. L'assurance de base obligatoire doit prendre en charge pendant une durée limitée les frais des médicaments qui améliorent nettement les chances de succès d'un arrêt du tabagisme. 


\section{Eloigner les jeunes de la consommation} de tabac

La cigarette est le seul produit mis à disposition légalement alors que, consommé selon les indications du fabricant, il entraîne la mort prématurée de la moitié de ses consommateurs. C'est pourquoi nous revendiquons l'interdiction de la publicité pour le tabac. Pour l'instant, les fabricants de cigarettes peuvent faire de la publicité et de la promotion pour leurs produits sans grande restriction en Suisse.

Le prix élevé des cigarettes entraîne un recul de la consommation, notamment chez les jeunes. Mais les cigarettes sont encore trop bon marché dans notre pays par rapport à nos voisins.

Il faut aussi rendre plus difficile l'accès, des jeunes en particulier, aux produits du tabac. Il est plus facile d'acheter des cigarettes que du pain ou du lait.

Les établissements de formation ont une mission importante de promotion de la santé. Des écoles sans tabac peuvent renforcer les efforts dans ce sens.

\section{Opposition ferme à l'industrie du tabac}

La convention-cadre de l'OMS pour la lutte antitabac, que la Suisse a signé en juin 04, crée les bases d'une mise en œuvre nationale des revendications proposées:

- lieux publics sans fumée;

- protection contre la fumée passive, y compris sur les lieux de travail;

- soutien en cas d'arrêt du tabagisme;

- prix élevé des cigarettes;

- interdiction de la publicité pour le tabac;

- restriction de l'accès aux produits du tabac, notamment pour les jeunes;

- écoles sans tabac.

Nous invitons le Conseil fédéral et le Parlement à ratifier sans délai la convention-cadre de l'OMS pour la lutte anti-tabac pendant la législature en cours. Une réglementation stricte des produits du tabac est nécessaire pour renforcer la promotion d'une vie sans tabac. 